\title{
The ACTyourCHANGE in Teens Study Protocol: An Acceptance and Commitment Therapy-Based Intervention for Adolescents with Obesity: A Randomized Controlled Trial
}

\author{
Anna Guerrini Usubini ${ }^{1,2, *}$, Roberto Cattivelli ${ }^{1,2}$, Vanessa Bertuzzi ${ }^{2}{ }^{\circledR}$, Giorgia Varallo ${ }^{1,2}$, \\ Alessandro Alberto Rossi ${ }^{3,4}{ }^{\circledR}$, Clarissa Volpi ${ }^{1}$, Michela Bottacchi ${ }^{1}$, Sofia Tamini ${ }^{5}{ }^{\infty}$, Alessandra De Col ${ }^{5}$, \\ Giada Pietrabissa ${ }^{1,2}$, Stefania Mannarini ${ }^{3,4} \mathbb{D}^{-}$, Gianluca Castelnuovo ${ }^{1,2}$, Enrico Molinari ${ }^{1,2}$ \\ and Alessandro Sartorio ${ }^{5,6}{ }^{(1)}$
}

1 Istituto Auxologico Italiano IRCCS, Psychology Research Laboratory, 20145 Milan, Italy; r.cattivelli@auxologico.it (R.C.); g.varallo@auxologico.it (G.V.); c.volpi@auxologico.it (C.V.); m.bottacchi@auxologico.it (M.B.); g.pietrabissa@auxologico.it (G.P.); gianluca.castelnuovo@auxologico.it (G.C.); molinari@auxologico.it (E.M.)

2 Department of Psychology, Catholic University of Milan, 20123 Milan, Italy; vanessa.bertuzzi@unicatt.it

3 Department of Philosophy, Sociology, Education, and Applied Psychology, Section of Applied Psychology, University of Padova, 35139 Padova, Italy; a.rossi@unipd.it (A.A.R.); stefania.mannarini@unipd.it (S.M.)

check for updates

Citation: Guerrini Usubini, A. Cattivelli, R.; Bertuzzi, V.; Varallo, G.; Rossi, A.A.; Volpi, C.; Bottacchi, M.; Tamini, S.; De Col, A.; Pietrabissa, G.; et al. The ACTyourCHANGE in Teens Study Protocol: An Acceptance and Commitment Therapy-Based Intervention for Adolescents with Obesity: A Randomized Controlled Trial. Int. J. Environ. Res. Public Health 2021, 18, 6225. https://doi.org/ 10.3390/ijerph18126225

Academic Editors: Joemer Maravilla and Natalie Thomas

Received: 19 April 2021

Accepted: 4 June 2021

Published: 9 June 2021

Publisher's Note: MDPI stays neutral with regard to jurisdictional claims in published maps and institutional affiliations.

Copyright: (c) 2021 by the authors. Licensee MDPI, Basel, Switzerland. This article is an open access article distributed under the terms and conditions of the Creative Commons Attribution (CC BY) license (https:// creativecommons.org/licenses/by/ $4.0 /)$.
4 Interdepartmental Center for Family Research, University of Padova, 35139 Padova, Italy

5 Istituto Auxologico Italiano, IRCCS, Experimental Laboratory for Auxo-Endocrinological Research, 28824 Piancavallo (VB), Italy; s.tamini@auxologico.it (S.T.); a.decol@auxologico.it (A.D.C.); sartorio@auxologico.it (A.S.)

6 Istituto Auxologico Italiano, IRCCS, Division of Auxology, 28824 Piancavallo (VB), Italy

* Correspondence: u.guerrini@auxologico.it

Abstract: This Randomized Controlled Trial [(RCT) aims to evaluate the effectiveness of a brief Acceptance and Commitment Therapy (ACT)-based intervention combined with treatment as usual (TAU) compared to TAU only in improving psychological conditions in a sample of adolescents with obesity (body mass index, BMI > 97th percentile for age and sex) within the context of a wider multidisciplinary rehabilitation program for weight loss. Fifty consecutive adolescents (12-17 years) of both genders with obesity will be recruited among the patients hospitalized in a clinical center for obesity rehabilitation and randomly allocated into two experimental conditions: ACT + TAU vs. TAU only. Both groups will attend a three-week in-hospital multidisciplinary rehabilitation program for weight loss. The ACT + TAU condition comprises a psychological intervention based on ACT combined with a standard psychological assessment and support to the hospitalization. The TAU comprises the standard psychological assessment and support to the hospitalization. At pre- to post-psychological intervention, participants will complete the Avoidance and Fusion Questionnaire for Youth, the Psychological Well-Being Scale, the Depression Anxiety Stress Scale, the Difficulties in Emotion Regulation Scale, and the Emotional Eating subscale of the Dutch Eating Behavior Questionnaire to assess psychological well-being as the primary outcome and experiential avoidance, psychological distress, emotional dysregulation, and emotional eating as secondary outcomes. Repeated-measures ANOVAs $(2 \times 2)$ will be conducted. The study will assess the effectiveness of a brief ACT-based intervention for adolescents with obesity in improving their psychological conditions by targeting specific core processes of the ACT framework (openness, awareness, and engagement). Future directions of the study will assess whether these psychological processes will contribute to addressing long-term weight loss.

Keywords: obesity rehabilitation; adolescents; acceptance and commitment therapy; psychological well-being; psychological flexibility 


\section{Introduction}

Obesity in childhood and adolescence is becoming a major public health concern. In this respect, recent estimates pointed out that globally, 124 million children and adolescents, aged between 5 and 19 years, were obese [1] In Europe, the prevalence of overweight and obese children and adolescents aged between 5 and 19 years is reported around 19\%, with a higher prevalence in southern European countries [2].

Obesity in children and adolescents is associated with a higher risk of developing several physical and psychological problems that have an impact on their emotional development [3]. Research has reported associations between childhood and adolescent obesity and some form of psychopathology, such as depression [4], anxiety, and attentiondeficit/hyperactivity disorder [5]. Children and adolescents with obesity are more likely to suffer from psychosocial disadvantages such as discrimination, social isolation, and bullying because of their weight [6-9]. Moreover, obesity in childhood and adolescence is associated with poor body image, low self-esteem [10], and low quality of life and well-being [11].

This evidence suggests that obesity is a complex disease that may dramatically impair psychological health, representing a risk factor for children and adolescents' future development. This aspect calls for a better understanding of the associated risk factors and requires a multidisciplinary approach —entailing medical, nutritional, physical, and psychological components-aimed to address not only weight loss but also improving psychological conditions and treating obesity-related comorbidities [12,13].

The development of dysfunctional eating habits is one of the main factors linked to body weight, and it can be considered a barrier to weight loss [14,15]. Evidence from the literature suggests that unhealthy and dysfunctional eating habits could be related to a failure in emotional regulation strategies that leads individuals to eat in response to difficult emotions, as in the case of emotional eating defined as the tendency to eat in response to negative feelings [16].

Emotional regulation is defined as the ability to manage emotions, which means to recognize, understand, accept, and modulate flexible responses to emotions [17]. High emotion regulation allows individuals to act accordingly with personal goals, even in the presence of difficult emotions, while controlling for impulsive behaviors. On the contrary, when emotional regulation is lacking, emotional eating is undertaken to regulate difficult feelings.

Since emotional eating was related to higher consumption of sweet and fatty food, as an attempt to regulate negative feelings, it was found to be positively associated with weight gain and obesity, both in adults [18] and in younger populations [19].

A theoretical model [20] for explaining disordered eating suggests that momentary stimuli such as the availability of tasty food in the environment, in combination with a modern sedentary lifestyle, accompanied by difficult emotions, can lead people to move toward behaviors perceived to increase or maintain a hedonic state of pleasure, such as eating tasty food immediately. The model also suggests that values clarity and behavioral commitment [to connect healthy eating habits to important life values], awareness [of their automatic thoughts], and distress tolerance [negative internal states] are specific self-regulation skills that can help individuals to deal with difficult emotions and control for their behaviors. Such skills are the main focus of Acceptance and Commitment Therapy.

Acceptance and Commitment Therapy (ACT) [21] is a third-wave CBT raised in the last twenty years. ACT aims to promote psychological flexibility, which is defined as the ability to live in the "here and now" current moment consciously while implementing behaviors to live in line with one's values. This can be seen in opposition to experiential avoidance (or psychological inflexibility), which is the attempt to avoid unpleasant internal states, such as thoughts, feelings, and bodily sensations. Psychological flexibility is an important component for health promotion [22], since it was found that it helps to commit to behaviors that are coherent with deeply held values and a life directions that a person chooses as important and meaningful in his life [23]. Concurrently, it entails staying in contact with the 
present moment, regardless of unpleasant internal states, such as thoughts and emotions, which are considered as a normal part of human life. By shifting the focus from efforts to change dysfunctional thoughts and feelings, in ACT, the emphasis is placed on promoting behaviors that are consistent with personal values and goals even when dysfunctional thoughts and feeling are present, by promoting acceptance and awareness of personal internal states. The promotion of psychological flexibility is based on three pillars: openness, awareness, and engagement. Openness refers to the willingness to develop an open and acceptable attitude toward one's personal internal states such as thoughts, emotions, and bodily sensations; awareness refers to the ability to act intentionally with awareness about personal thoughts and sensations, without automatically reacting; engagement refers to engage oneself in committed behaviors related to personal values-that is, chosen life directions [24].

Several ACT-based protocols have been successfully applied to adolescents targeting a broad range of conditions, such as cystic fibrosis [25], chronic pain [26], ADHD [27], and at-risk adolescents [28]. In a pilot study conducted by Hayes, Boyd, and Sewell [29] thirty adolescents referred to a psychiatric outpatient service were randomly assigned to two conditions: in one, they received an ACT intervention; in the other, participants received a standard intervention for depression. The results showed a significant decrease in depressive symptoms in patients undergoing the ACT intervention. In a study conducted by Kemani and colleagues [30], an ACT-based intervention was applied to adolescents with chronic pain and their family members. Results showed improved acceptance of chronic pain and psychological flexibility in adolescents as well as decreased depressive symptoms in their parents. ACT has also been applied to adolescents with post-traumatic stress disorder [31], proving a reduction of symptoms after a 10-week intervention.

Unfortunately, there are not many studies in the literature concerning the application of an ACT protocol for adolescents with overweight/obesity problems. However, evidence suggests that psychological flexibility is an important key factor of psychological health, since it allows people to engage in meaningful challenges, following a person's self-concept and important life domains [22]. To the best of our knowledge, only one pilot study published in 2019 [32], evaluating the effectiveness of an ACT-based psychological intervention applied in combination with a 16-week lifestyle behavioral modification program in a sample of obese adolescents, was found. This study showed that the intervention was effective in promoting a reduction in BMI and an overall improvement in physical activity and psychological status of obese adolescents. Furthermore, the intervention was positively evaluated by both the adolescents and their parents who participated in the study.

Given these premises, the present study aims to evaluate the effectiveness of a brief ACT-based psychological intervention, compared to TAU in improving psychological conditions in a sample of adolescents with obesity attending a multidisciplinary rehabilitation program for weight loss.

\section{Materials and Methods}

\subsection{Study Design}

A superiority Randomized Controlled Trial (RCT) with parallel groups will be conducted with an ACT-based intervention plus TAU compared with TAU only for adolescents with obesity attending a multidisciplinary rehabilitation program for weight loss.

\subsection{Participants}

Participants will be consecutively recruited at the admission to the division of Auxology, Istituto Auxologico Italiano IRCCS, Piancavallo (VB), which is located in the NorthWest of Italy, a specialized clinical center [i.e., third level] for weight loss and obesity rehabilitation.

Participants of both genders will be eligible for the study if they meet the following criteria: (1) aged between 12 and 17 according to the classification of the pubertal status [33]; (2) BMI > 97th centile according to age- and sex-specific Italian charts [34]; (3) Italian mother 
tongue; (4) written and informed consent to participate from both parents and written assent from participants. Patients will be excluded if they have any physical or psychiatric problems according to the Diagnostic and Statistical Manual of Mental Disorders (DSM-5) criteria that could compromise participation in the study.

\subsection{Measures}

All demographical (gender, age, nationality, educational level, and family composition) and clinical data will be collected via self-report using Italian validated and widely used questionnaires. The following psychological measures will be collected pre (Time $0 /$ week 1 ) and post (Time 1/week 3) psychological intervention.

\subsubsection{Primary Outcomes}

Psychological Well-Being. The Psychological Well-Being Scales (PWB) [35] will be administered to assess psychological well-being. It is a self-report measure that explores six dimensions: self-acceptance, positive relationships with others, autonomy, environmental control, personal growth, and life purpose. The questionnaire consists of 18 items rated on a 4-point Likert scale ranging from 1 ("completely disagree") to 4 ("completely agree"). The Italian version [36], assessed for Italian adolescents [37], is reported to have good psychometric properties (test-retest correlation coefficients: Self-acceptance: $r=0.82$; Positive relationships: $r=0.81$; Autonomy: $r=0.21$; Environmental control: $r=0.31$; Life purpose: $r=0.81$; Personal growth: $r=0.78$ ).

\subsubsection{Secondary Outcomes}

Experiential avoidance and fusion. The Avoidance and Fusion Questionnaire for Youth (AFQ-Y) [38] will be administered as a measure of experiential avoidance and fusion in adolescents. It consists of 8 items rated on a 5-point Likert scale ranging from 0 ["not at all true"] to 4 ["absolutely true"]. The Italian version [39] is reported to have moderate internal consistency (Cronbach's alpha $=0.69)$ and good test-retest reliability $(r=0.64)$.

Psychological distress. The Depression Anxiety Stress Scale (DASS-21) [40] will be administered as a measure of psychological distress, which is widely used in samples of adolescents [41,42]. It is a self-report instrument that measures several negative internal states: depression, anxiety, and stress. It consists of 21 items rated on a 4-point Likert scale, ranging from 0 to 3 . The Italian version [43] showed good psychometric properties (Cronbach's alpha values of subscales ranged from 0.83 to 0.91 . The Cronbach's alpha of the total score was $=0.92$ ).

Emotional dysregulation. The Difficulties in Emotion Regulation Scale (DERS) [17] will be administered to assess difficulties in emotional dysregulation. This is a self-report questionnaire consisting of 36 items rated on a 5-point Likert scale ranging from 1 ("almost never") to 5 ("almost always"), which explores the following subscales: non-acceptance of negative emotions, inability to undertake purposeful behavior when experiencing negative emotions, difficulty in controlling impulsive behavior when experiencing negative emotions, limited access to emotion regulation strategies that are considered effective, lack of awareness of one's emotions, and lack of understanding of the nature of one's emotional responses. The Italian version [44], which is widely used in samples of adolescents [45,46], is reported to have good psychometric properties (Cronbach's alpha values ranged from 0.77 to 0.89 . The Cronbach's alpha of the total score was 0.92).

Emotional eating. The Emotional Eating subscale of the Dutch Eating Behavior Questionnaire (DEBQ) [47] will be administered to assess emotional eating. The DEBQ is a self-report questionnaire used to detect eating behaviors. The Emotional Eating subscale consists of 13 items, rated on a 5-step Likert scale ranging from 0 ("never") to 4 ("almost always"). The Italian version [48] showed good psychometric properties (Cronbach's alpha $=0.97$ ). The DEBQ was chosen as a measure of emotional eating in our sample, since no other validated instruments are available, except for the DEBQ 
version for parents [49], which is unfortunately unsuitable for parents of our sample of hospitalized adolescents.

\subsection{Randomization Procedure}

Participants will be randomly assigned to the experimental (ACT+ TAU) or control (TAU) group. We will perform simple randomization with a 1:1 allocation ratio using the web site Randomization.com (http: / / www.randomization.com) (accessed on 8 June 2021). Randomization will occur after the baseline measurements in order to ensure similar baseline characteristics of groups.

Allocation concealment will be ensured, since all the patients will generate an anonymous code that will be associated with the randomization generated by the program. Researchers will be blind to the association made.

\subsection{Procedures}

The trial will be conducted within the context of a three-week in-hospital multidisciplinary rehabilitation program for weight loss based on nutritional, physical, and psychological rehabilitation [50-52], according to the Italian National Health System recommendations. As part of the nutritional program, adolescents will be assessed by a staff dietician and placed on an individualized hypocaloric diet composed of $53 \%$ carbohydrates, $26 \%$ fat, and $21 \%$ protein [53] and a fluid intake of at least $1500 \mathrm{~mL}$ per day ${ }^{-1}$. They will also follow a daily nutritional counseling program provided both in individual and group settings, comprising dietetics lessons. As for physical rehabilitation, young patients will participate in a physical activity program consisting of five group training sessions per week lasting about 45-60 min per day of physical therapy comprising aerobic activities such as walking and cycling under the supervision of a therapist. The physical program also entails $2 \mathrm{~h}$ per week of aerobic leisure activities. As far as the psychological component is concerned, patients will take part in psychological counseling sessions that are provided at least once a week lasting about one hour each, carried out by a clinical psychologist, and aimed at promoting a healthy lifestyle and addressing psychological factors related to the onset of dysfunctional lifestyle habits.

At week 1 of the hospitalization, all the patients will be screened for admission to the study with a clinical interview conducted by a clinical psychologist blinded to research aims to provide information about the study and assess the eligibility criteria according to the Diagnostic and Statistical Manual of Mental Disorders (DSM-5). Weight and height, to calculate BMI, will be measured by the internal medical team. Once we obtain informed consent to participate from parents and written assent from participants, young patients recruited for the study will be randomly assigned into two conditions. The experimental group will attend TAU plus a brief ACT-based intervention that comprises three sessions, provided once a week, lasting about one hour each, while the control group will attend TAU only.

At pre (Time 0/week 1 ) and post-psychological intervention (Time 1/week 3 ), patients of both conditions will be invited to fill the questionnaires under the supervision of a member of the research team.

In case of any form of psychological discomfort due to the participation in the intervention or any doubt or need for information concerning the trial, participants will have the opportunity to consult the psychologist responsible for the study. Once enrolled, patients can withdraw from the study at any time. This will not affect their future treatment.

The clinical psychologist who will conduct the sessions, participants, and observers will be blinded to research aims.

Completed questionnaires will be stored in a room used for research in the hospital, which is accessible only for research team members. Data will be stored on passwordprotected files kept for five years after the end of the trial.

The study protocol was approved by the Ethical Committee of the Istituto Auxologico Italiano (registration number 2021_01_26_03) and registered on ClinicalTrials.gov (last up- 
date on 7 June 2021). All procedures will be conducted following the Helsinki Declaration and its later advancements.

The schedule of enrolment, intervention, and assessment in the study is presented in Figure 1 (see also Supplementary Materials).

\begin{tabular}{|c|c|c|c|c|c|c|}
\hline & Enrolment & Pre-Intervention & \multicolumn{3}{|c|}{ Intervention } & \multirow{2}{*}{$\begin{array}{c}\text { Post-Intervention } \\
\text { T1 } \\
\text { (Week 3) }\end{array}$} \\
\hline TIMEPOINT & Week 1 & $\begin{array}{c}\text { T0 } \\
\text { (week 1) }\end{array}$ & Week 1 & Week 2 & Week 3 & \\
\hline \multicolumn{7}{|l|}{ ENROLMENT: } \\
\hline Eligibility screen & $\mathrm{x}$ & & & & & \\
\hline Informed consent & $\mathrm{x}$ & & & & & \\
\hline Allocation & & $\mathrm{x}$ & & & & \\
\hline \multicolumn{7}{|l|}{ INTERVENTION: } \\
\hline \multicolumn{7}{|l|}{$\mathrm{ACT}+\mathrm{TAU}$} \\
\hline \multicolumn{7}{|l|}{ TAU only } \\
\hline \multicolumn{7}{|l|}{ ASSESSMENTS: } \\
\hline $\begin{array}{r}\text { Demographical data } \\
A F Q-Y \\
P W B \\
D A S S-21 \\
D E R S \\
D E B Q \_E E\end{array}$ & & $\mathrm{x}$ & & & & $\mathrm{x}$ \\
\hline
\end{tabular}

Figure 1. Spirit figure.

\subsection{The ACT-Based Intervention}

Participants assigned to the ACT group will attend treatment as usual plus a brief ACT-based intervention comprising three sessions, provided once a week, lasting about one hour each. The proposed intervention was designed by the authors of the study. It was developed following the main ACT-based manuals with adjustments according to the users and the context of the study implementation [54-56]. The ACT-based intervention is aimed to promote acceptance, awareness, and engagement in valued behaviors, as alternatives to deal with dysfunctional thoughts and feelings. Experiential exercises and key metaphors will be used to target the core processes of the intervention.

Specifically, we developed a practical and interactive intervention, which comprises practical activities such as roleplay and writing activities and imaginative activities supported by metaphors. The sessions are based on the use of age-appropriate language to convey the most complex therapeutic processes easily. According to the model of Strosahl and colleagues [57], sessions will respectively target the components of openness, awareness, and engagement. In the Openness session, young patients are guided to recognize distressing thoughts, emotions, or body sensations they are dealing with and imagine what their life would be like without their source of suffering. Then, they are invited to recognize their ineffective strategies they use for controlling or avoid pain instead of working on growing life. In the Awareness section, young patients are guided to learn how to feel in contact with the present internal states at the moment they are and just note what they are experiencing, without reacting. They are also invited to consider the self as a viewpoint, or a psychological space from which observing their thoughts and feelings without being overwhelmed. Finally, in the Engagement session, young patients are asked to list the personal qualities they have or those they desire to have for themselves. They are also guided to clarify their values, as chosen life direction they desire to pursue to become the person they want to be. Then, they are required to identify which behaviors need to be engaged in order to achieve their values. 
A detailed description of the sessions is provided in Table 1.

Table 1. The ACT-based intervention.

\begin{tabular}{|c|c|c|c|}
\hline $\begin{array}{l}\text { Session } \\
\text { Number }\end{array}$ & Domains & Goals and Therapeutic Processes & Experiential Activities and Metaphors \\
\hline $\begin{array}{c}\text { Session } 1 \\
\text { Week } 1\end{array}$ & Openness & $\begin{array}{l}\text { The purpose of this session is to develop } \\
\text { the willingness to experiencing distress } \\
\text { and undesirable private events as part of } \\
\text { human experience, without judgment or } \\
\text { attempts to avoid or control internal } \\
\text { states, even if they are unpleasant. }\end{array}$ & $\begin{array}{l}\text { Experiential exercise: “How would my life } \\
\text { be if ..." } \\
\text { In this experiential activity, young patients are } \\
\text { guided to answer the key question: "How would } \\
\text { my life be if I didn't have ... [my problem that I } \\
\text { have]". The problem could be their weight or } \\
\text { anything else related to their condition of obesity } \\
\text { or any other perceived problem in their life. } \\
\text { Participants are encouraged to describe what } \\
\text { they desire to do if they did not have obesity, in } \\
\text { order to take distance, observe and accept their } \\
\text { conditions and related thoughts and feelings. }\end{array}$ \\
\hline $\begin{array}{c}\text { Session } 2 \\
\text { Week } 2\end{array}$ & Awareness & $\begin{array}{l}\text { The purpose of this session is to promote } \\
\text { the ability to be present at the moment } \\
\text { and face events as contextually situated. } \\
\text { To be aware means stepping back from } \\
\text { suffering situations and seeing them in } \\
\text { the context where they occur. }\end{array}$ & $\begin{array}{l}\text { Metaphor: "The sky and weather metaphor" [58]. } \\
\text { In this metaphor, personal thoughts and feelings } \\
\text { are presented as the weather and the self as the } \\
\text { sky. The weather naturally changes. Despite that, } \\
\text { it can never harm or change the sky. No matter } \\
\text { how bad the weather, the sky always has room } \\
\text { for it. Sometimes, we forget that the sky is there, } \\
\text { but it is still there. In the same way, difficult } \\
\text { thoughts and feelings can occur. No matter how } \\
\text { harmful they are, the self is still there. We can } \\
\text { always learn how to access this part of us. It is a } \\
\text { safe space that contains difficult thought } \\
\text { and feelings. }\end{array}$ \\
\hline
\end{tabular}

The purpose of this session is to foster values clarification and engagement in actions linked to personal values, such as Session 3 Week 3
Engagement relationships and personal growth. If a person engages himself in committed actions driven by chosen life directions, they can pursue a meaningful and coherent life.
Experiential exercise: “The treasure hunt". In this experiential exercise, young patients are asked to draw a treasure hunt in which the treasure is a value in their life and the route is made of behaviors that need to be engaged to reach a meaningful life.

\subsection{Treatment Fidelity}

The research group comprises licensed psychologists, researchers, and doctoral students with expertise in clinical interventions in health care settings and research.

Sessions will be administered by a licensed clinical psychologist with about three years of expertise in ACT clinical practice for adolescents both in individual and group settings blinded to research aims.

The structure and the content of the sessions are consistent with ACT theory, as mentioned above.

To ensure that the intervention will be delivered as planned, direct observation of the intervention is guaranteed. In line with previous studies [59] two independent bachelorlevel observers blinded to research aims will observe at least $20 \%$ of sessions to evaluate their adherence to the protocol, after a period of training. By using a checklist, they will assess the coverage of the intervention's components. Checklists include all the contents and the experiential exercises planned for each session. Coders have to achieve a minimum of $80 \%$ reliability with the expert trainers and each other. With a lower level of agreement, the data will be dismissed. 


\subsection{Sample Size Calculation}

In line with previous studies $[60,61]$ to calculate the sample size, an a priori power analysis was carried out using the software G.Power 3.1.9.4 [62].

The required sample size was computed for an analysis of variance (ANOVA) with 2 groups (between variable; ACT + TAU vs. TAU) $\times 2$ times (within variable; Time $0 /$ Time 1 ). To the best of the author's knowledge, no other similar studies are available in the literature. Therefore, the a priori effect size (Cohen's $f$ ) for the global effect (groups $x$ time) was set to 0.2 (small effect size) [63]. Moreover, the Type I error [ $\alpha$ ] probability was set to 0.05 , and the power [1- $\beta$ ] was set to 0.95 . In addition, correlation among repeated measures to 0.3 (small-moderate correlation/linear effect). Non-sphericity was assumed, and it was set to 1 . Results showed that there is a more than $95 \%$ chance of correctly rejecting the null hypothesis of no significant effect of the interaction with an overall sample of 116 subjects: a minimum of 58 participants per group.

\subsection{Statistical Analysis}

Descriptive statistical analyses will be conducted to investigate the baseline characteristics of the sample. To evaluate differences between the two groups (ACT + TAU vs. TAU only) across the two time points (pre-Time 0 /week 1 ; post-Time 1/week 3 psychological intervention), repeated measure between groups ANOVAs will be performed for each dependent variable - namely, AFQ-Y, PWB, DASS-21, DERS, and DEBQ. In this case, a global effect size (Cohen's $f$ and/or eta-squared $-\eta^{2}$ ) will be used to quantify the [global] difference of the two groups across times. Global effects size will be interpreted with the following benchmarks [63]: null $\left(f<0.10 ; \eta^{2}<0.003\right.$ ); small (f from 0.10 to $0.25 ; \eta^{2}$ from 0.003 to 0.039 ); moderate ( $f$ from 0.25 to $0.40 ; \eta^{2}$ from 0.40 to 0.110 ); and large ( $f \geq 0.40$; $\eta^{2}>0.110$ ). Moreover, several focused comparisons will be performed to assess differences both within and between groups. In particular, differences between the two groups will be assessed using an independent samples t-test both at the baseline as well as at the end of psychological treatment, separately-namely, T1: ACT + TAU vs. TAU and T2: ACT + TAU vs. TAU. Conversely, differences within each group across the two time points will be assessed using dependent samples t-test-namely, ACT + TAU: T1 vs. T2; and TAU: T1 vs. T2. The magnitude of the differences will be interpreted by using the Cohen's d [64] — more in detail, for between-groups comparisons, the classical Cohen's d formula will be used; in contrast, for within-groups comparisons, the modified formula for dependent data will be used. Each Cohen's d will be interpreted using the following benchmarks: null $(d<0.20)$, small ( $\mathrm{d}$ from 0.20 to 0.49 ), moderate ( $\mathrm{d}$ from 0.50 to 0.79 ), and strong $(d>0.80)$.

Participants who reported missing data as well as subjects who will not participate in each therapy session will be excluded from the final sample, and thus, their data will not be analyzed. Moreover, also, participants who drop out from the program will be excluded from the final sample.

Analyses will be carried out using Jamovi (version 1.6.15) [64].

\section{Discussion}

This work is aimed at describing the study that will be implemented to evaluate the efficacy of a brief ACT-based intervention in improving psychological conditions of youth with obesity within a context of a multidisciplinary in-hospital obesity rehabilitation programs.

The dramatic global increase of childhood and adolescence obesity calls for a better understanding of the associated risk factors and requires effective treatments. Multidisciplinary interventions targeting healthy diet and physical activity are needed to treat obesity effectively [65]. However, since obesity impairs both the physical and psychological health of children and adolescents, one of the key focuses of the interventions should be psychological well-being and mental health.

The majority of treatments are substantially conceived to prevent and control the widespread increase of childhood and adolescence obesity through parental education on proper nutritional requirements for their children and the implementation of healthy 
eating habits and physical activity [66]. Conversely, few studies have been implemented to provide psychological interventions, targeting emotional regulation in children and adolescents, which is essential for healthy psychological functioning [67] and healthy eating habits. In this regard, the present study has been conceived.

The psychological intervention proposed in the current study will be implemented within a multidisciplinary in-hospital program for weight loss and obesity rehabilitation. Even if the high specificity of the context could represent a limitation for the generalizability of potential significant results, an inpatient rehabilitative program allows young people to live in an environment where they may eat healthily and engage in physical activity. This could be the setting of choice to improve psychological well-being and important self-regulation skills related to healthy habits in adolescents with obesity, according to the ACT framework.

Consistent results of this study would be able to open promising directions for future research. Specifically, by incorporating measurement of weight loss and planning a followup over time after the rehabilitation program, it will be possible to assess whether the intervention could be helpful to sustain long-term weight loss and long-term adoption of a healthy lifestyle.

\section{Conclusions}

Significant results from this study would provide additional information concerning how ACT can enhance the psychological conditions of adolescents with obesity, seeking treatment for weight loss and rehabilitation.

Supplementary Materials: The following are available online at https:/ / www.mdpi.com/article/10 .3390/ijerph18126225/s1, Table S1: Spirit Checklist.

Author Contributions: Conceptualization, A.G.U. and R.C.; methodology, A.A.R. and G.P.; writingoriginal draft preparation, A.G.U., R.C., V.B., G.V., C.V., M.B., S.T. and A.D.C.; writing-review and editing, G.C., E.M., S.M. and A.S.; supervision, A.S.; project administration, A.G.U. All authors have read and agreed to the published version of the manuscript.

Funding: This research received no external funding.

Institutional Review Board Statement: The study was conducted according to the guidelines of the Declaration of Helsinki, and approved by the Ethics Committee of ISTITUTO AUXOLOGICO ITALIANO (protocol code 2021_01_26_03).

Informed Consent Statement: Informed consent will be obtained from all subjects involved in the study.

Data Availability Statement: The collected in this study will be available on request from author A.G.U. with the permission of author A.S. The data will not be publicly available due to privacy/ethical restrictions.

Conflicts of Interest: The authors declare no conflict of interest.

\section{References}

1. Bentham, J.; Di Cesare, M.; Bilano, V.; Bixby, H.; Zhou, B.; Stevens, G.A.; Riley, L.M.; Taddei, C.; Hajifathalian, K.; Lu, Y.; et al. Worldwide trends in body-mass index, underweight, overweight, and obesity from 1975 to 2016: A pooled analysis of 2416 population-based measurement studies in 128.9 million children, adolescents, and adults. Lancet 2017, 390, 2627-2642. [CrossRef]

2. World Health Organization (WHO). Obesity and Overweight; WHO: Geneva, Switzerland, 2017; Available online: http://www. who.int/mediacentre/factsheets/fs311/en/ (accessed on 25 October 2017).

3. Sagar, R.; Gupta, T. Psychological Aspects of Obesity in Children and Adolescents. Indian J. Pediatr. 2018, 85, 554-559. [CrossRef]

4. Quek, Y.H.; Tam, W.W.S.; Zhang, M.W.B.; Ho, R.C.M. Exploring the association between childhood and adolescent obesity and depression: A meta-analysis. Obes. Rev. 2017, 18, 742-754. [CrossRef]

5. Waring, M.E.; Lapane, K.L. Overweight in children and adolescents in relation to attention-deficit/ hyperactivity disorder: Results from a national sample. Pediatrics 2008, 122. [CrossRef]

6. Morrison, K.M.; Shin, S.; Tarnopolsky, M.; Taylor, V.H. Association of depression \& health related quality of life with body composition in children and youth with obesity. J. Affect. Disord. 2015, 172, 18-23. [CrossRef] [PubMed]

7. Beck, A.R. Psychosocial Aspects of Obesity. NASN Sch. Nurse 2016, 31, 23-27. [CrossRef] 
8. Halfon, N.; Larson, K.; Slusser, W. Associations between obesity and comorbid mental health, developmental, and physical health conditions in a nationally representative sample of us children aged 10 to 17. Acad. Pediatr. 2013, 13, 6-13. [CrossRef]

9. Rankin, J.; Matthews, L.; Cobley, S.; Han, A.; Sanders, R.; Wiltshire, H.D.; Baker, J.S. Psychological consequences of childhood obesity: Psychiatric comorbidity and prevention. Adolesc. Health Med. Ther. 2016, 7, 125-146. [CrossRef]

10. Wang, F.; Veugelers, P.J. Self-esteem and cognitive development in the era of the childhood obesity epidemic. Obes. Rev. 2008, 9 615-623. [CrossRef]

11. Anderson, Y.C.; Wynter, L.E.; Treves, K.F.; Grant, C.C.; Stewart, J.M.; Cave, T.L.; Wouldes, T.A.; Derraik, J.G.B.; Cutfield, W.S.; Hofman, P.L. Assessment of health-related quality of life and psychological well-being of children and adolescents with obesity enrolled in a New Zealand community-based intervention programme: An observational study. BMJ Open 2017, 7, e015776. [CrossRef]

12. Carter, F.A.; Jansen, A. Improving psychological treatment for obesity. Which eating behaviours should we target? Appetite 2012, 58, 1063-1069. [CrossRef]

13. Giusti, E.M.; Spatola, C.; Brunani, A.; Kumbhare, D.; Oral, A.; Ilieva, E.; Kiekens, C.; Pietrabissa, G.; Manzoni, G.M.; Imamura, M.; et al. ISPRM/ESPRM Guidelines on Physical and Rehabilitation Medicine (PRM) professional practice for adults with obesity and related comorbidities. Eur. J. Phys. Rehabil. Med. 2020, 56, 496-507. [CrossRef]

14. Keller, C.; Siegrist, M. Ambivalence toward palatable food and emotional eating predict weight fluctuations. Results of a longitudinal study with four waves. Appetite 2015, 85, 138-145. [CrossRef]

15. Sainsbury, K.; Evans, E.H.; Pedersen, S.; Marques, M.M.; Teixeira, P.J.; Lähteenmäki, L.; Stubbs, R.J.; Heitmann, B.L.; Sniehotta, F.F. Attribution of weight regain to emotional reasons amongst European adults with overweight and obesity who regained weight following a weight loss attempt. Eat. Weight Disord. 2019, 24, 351-361. [CrossRef] [PubMed]

16. Frayn, M.; Knäuper, B. Emotional Eating and Weight in Adults: A Review. Curr. Psychol. 2018, 37, 924-933. [CrossRef]

17. Gratz, K.L.; Roemer, L. Multidimensional Assessment of Emotion Regulation and Dysregulation: Development, Factor Structure, and Initial Validation of the Difficulties in Emotion Regulation Scale. J. Psychopathol. Behav. Assess. 2004, 26, 41-54. [CrossRef]

18. Van Strien, T.; Peter Herman, C.; Verheijden, M.W. Eating style, overeating and weight gain. A prospective 2-year follow-up study in a representative Dutch sample. Appetite 2012, 59, 782-789. [CrossRef]

19. Braet, C.; Claus, L.; Goossens, L.; Moens, E.; Van Vlierberghe, L.; Soetens, B. Differences in eating style between overweight and normal-weight youngsters. J. Health Psychol. 2008, 13, 733-743. [CrossRef] [PubMed]

20. Forman, E.; Butryn, M. A new look at the science of weight control: How acceptance and commitment strategies can address the challenge of self-regulation. Appetite 2015. [CrossRef]

21. Hayes, S.C.; Luoma, J.B.; Bond, F.W.; Masuda, A.; Lillis, J. Acceptance and Commitment Therapy: Model, processes and outcomes. Behav. Res. Ther. 2006. [CrossRef]

22. Kashdan, T.B.; Rottenberg, J. Psychological flexibility as a fundamental aspect of health. Clin. Psychol. Rev. 2010, 30, 865-878. [CrossRef]

23. Lillis, J.; Niemeier, H.M.; Ross, K.M.; Graham Thomas, J.; Leahey, T.; Unick, J.; Kendra, K.E.; Wing, R.R. Weight loss intervention for individuals with high internal disinhibition: Design of the Acceptance Based Behavioral Intervention (ABBI) randomized controlled trial. BMC Psychol. 2015, 3, 17. [CrossRef]

24. Hayes, S.C.; Strosahl, K.D.; Wilson, K.G. Acceptance and Commitment Therapy: The Process and Practice of Mindful Change, 2nd ed.; The Guilford Press: New York, NY, USA, 2012; ISBN $9781609189624 / 9781609189648$.

25. Casier, A.; Goubert, L.; Theunis, M.; Huse, D.; De Baets, F.; Matthys, D.; Crombez, G. Acceptance and well-being in adolescents and young adults with cystic fibrosis: A prospective study. J. Pediatr. Psychol. 2011, 36, 476-487. [CrossRef]

26. Gauntlett-Gilbert, J.; Connell, H.; Clinch, J.; Mccracken, L.M. Acceptance and values-based treatment of adolescents with chronic pain: Outcomes and their relationship to acceptance. J. Pediatr. Psychol. 2013, 38, 72-81. [CrossRef]

27. Murrell, A.R.; Kapadia, V. Brief report on experiential avoidance and valuing in at-risk adolescents. Behav. Dev. Bull. 2011, 17, 38-42. [CrossRef]

28. Luciano, C.; Ruiz, F.J.; Torres, R.M.V.; Martín, V.S.; Martínez, O.G.; López, J.C.L. A relational frame analysis of defusion interactions in acceptance and commitment therapy. A preliminary and quasi-experimental study with at-risk adolescents. Int. J. Psychol. Psychol. Ther. 2011, 11, 165-182.

29. Hayes, L.; Boyd, C.P.; Sewell, J. Acceptance and Commitment Therapy for the Treatment of Adolescent Depression: A Pilot Study in a Psychiatric Outpatient Setting. Mindfulness 2011, 2, 86-94. [CrossRef]

30. Kemani, M.K.; Kanstrup, M.; Jordan, A.; Caes, L.; Gauntlett-Gilbert, J. Evaluation of an intensive interdisciplinary pain treatment based on acceptance and commitment therapy for adolescents with chronic pain and their parents: A nonrandomized clinical trial. J. Pediatr. Psychol. 2018, 43, 981-994. [CrossRef] [PubMed]

31. Woidneck, M.R.; Morrison, K.L.; Twohig, M.P. Acceptance and Commitment Therapy for the Treatment of Posttraumatic Stress Among Adolescents. Behav. Modif. 2014, 38, 451-476. [CrossRef]

32. Tronieri, J.S.; Wadden, T.A.; Leonard, S.M.; Berkowitz, R.I. A pilot study of acceptance-based behavioural weight loss for adolescents with obesity. Behav. Cogn. Psychother. 2019, 47, 686-696. [CrossRef] [PubMed]

33. Tanner, J. Foetus into Man: Physical Growth from Conception to Maturity; Harvard University Press: Cambridge, UK, 1990.

34. Cacciari, E.; Milani, S.; Balsamo, A.; Spada, E.; Bona, G.; Cavallo, L.; Cerutti, F.; Gargantini, L.; Greggio, N.; Tonini, G.; et al. Italian cross-sectional growth charts for height, weight and BMI (2 to 20 yr). J. Endocrinol. Investig. 2006, 29, 581-593. [CrossRef] 
35. Ryff, C.D. Happiness is everything, or is it? Explorations on the meaning of psychological well-being. J. Pers. Soc. Psychol. 1989, 57, 1069-1081. [CrossRef]

36. Ruini, C.; Ottolini, F.; Rafanelli, C.; Ryff, C.; Fava, G.A. La validazione italiana delle Psychological Well-being Scales (PWB). Riv. Psichiatr. 2003, 38, 117-130.

37. Sirigatti, S.; Stefanile, C.; Giannetti, E.; Iani, L.; Penzo, I.; Mazzeschi, A. Assessment of factor structure of Ryff's Psychological Well-Being Scales in Italian adolescents. Boll. Psicol. Appl. 2009, 259, 30-50.

38. Greco, L.A.; Lambert, W.; Baer, R.A. Psychological Inflexibility in Childhood and Adolescence: Development and Evaluation of the Avoidance and Fusion Questionnaire for Youth. Psychol. Assess. 2008, 20, 93-102. [CrossRef]

39. Schweiger, M.; Ristallo, A.; Oppo, A.; Pergolizzi, F.; Presti, G.; Moderato, P. Ragazzi in lotta con emozioni e pensieri: La validazione della versione italiana dell'avoidance and fusion questionnaire for youth (I-AFQ-Y). Psicoter. Cogn. Comport. 2017, 23, $141-162$.

40. Lovibond, S.; Lovibond, P. Manual for the Depression Anxiety Stress Scales; Psychology Foundation of Australia: Sydney, Australia, 1996.

41. Cosenza, M.; Ciccarelli, M.; Nigro, G. Decision-Making Styles, Negative Affectivity, and Cognitive Distortions in Adolescent Gambling. J. Gambl. Stud. 2019, 35, 517. [CrossRef]

42. Nigro, G.; Cosenza, M.; Ciccarelli, M. The blurred future of adolescent gamblers: Impulsivity, time horizon, and emotional distress. Front. Psychol. 2017, 8, 486. [CrossRef]

43. Bottesi, G.; Ghisi, M.; Altoè, G.; Conforti, E.; Melli, G.; Sica, C. The Italian version of the Depression Anxiety Stress Scales-21: Factor structure and psychometric properties on community and clinical samples. Compr. Psychiatry 2015, 60, 170-181. [CrossRef]

44. Giromini, L.; Velotti, P.; De Campora, G.; Bonalume, L.; Cesare Zavattini, G. Cultural Adaptation of the Difficulties in Emotion Regulation Scale: Reliability and Validity of an Italian Version. J. Clin. Psychol. 2012, 68, 989-1007. [CrossRef]

45. Somma, A.; Sharp, C.; Borroni, S.; Fossati, A. Borderline personality disorder features, emotion dysregulation and non-suicidal self-injury: Preliminary findings in a sample of community-dwelling Italian adolescents. Pers. Ment. Health 2017, 11, 23-32. [CrossRef] [PubMed]

46. Fossati, A.; Gratz, K.L.; Maffei, C.; Borroni, S. Impulsivity dimensions, emotion dysregulation, and borderline personality disorder features among Italian nonclinical adolescents. Bord. Personal. Disord. Emot. Dysregul. 2014, 1, 5. [CrossRef] [PubMed]

47. Van Strien, T.; Frijters, J.E.R.; Bergers, G.P.A.; Defares, P.B. The Dutch Eating Behavior Questionnaire (DEBQ) for assessment of restrained, emotional, and external eating behavior. Int. J. Eat. Disord. 1986, 5, 295-315. [CrossRef]

48. Dakanalis, A.; Zanetti, M.A.; Clerici, M.; Madeddu, F.; Riva, G.; Caccialanza, R. Italian version of the Dutch Eating Behavior Questionnaire. Psychometric proprieties and measurement invariance across sex, BMI-status and age. Appetite 2013, 71, 187-195. [CrossRef]

49. Caccialanza, R.; Nicholls, D.; Cena, H.; Maccarini, L.; Rezzani, C.; Antonioli, L.; Dieli, S.; Roggi, C. Validation of the Dutch Eating Behaviour Questionnaire parent version (DEBQ-P) in the Italian population: A screening tool to detect differences in eating behaviour among obese, overweight and normal-weight preadolescents. Eur. J. Clin. Nutr. 2004, 58, 1217-1222. [CrossRef]

50. Rigamonti, A.E.; Tringali, G.; De Micheli, R.; De Col, A.; Tamini, S.; Saezza, A.; Cella, S.G.; Sartorio, A. Impact of a three-week in-hospital multidisciplinary body weight reduction program on body composition, muscle performance and fatigue in a pediatric obese population with or without metabolic syndrome. Nutrients 2020, 12, 208. [CrossRef]

51. Lazzer, S.; Bravo, G.; Tringali, G.; De Micheli, R.; De Col, A.; Sartorio, A. A 3-Week Multidisciplinary Body Weight Reduction Program Improves Body Composition and Lower Limb Power Output in 3,778 Severely Obese Children and Adolescents. Front. Physiol. 2020, 11, 548. [CrossRef]

52. Pietrabissa, G.; Volpi, C.; Bottacchi, M.; Bertuzzi, V.; Guerrini Usubini, A.; Löffler-Stastka, H.; Prevendar, T.; Rapelli, G.; Cattivelli, R.; Castelnuovo, G.; et al. The impact of social isolation during the covid-19 pandemic on physical and mental health: The lived experience of adolescents with obesity and their caregivers. Int. J. Environ. Res. Public Health 2021, 18, 3026. [CrossRef]

53. Nutrition ISO. Recommended Levels of Energy and Nutrients Intake for the Italian Population (LARN); Edra Medical Publishing and New Media: Milano, Italy, 1996.

54. Halliburton, A.E.; Cooper, L.D. Applications and adaptations of Acceptance and Commitment Therapy (ACT) for adolescents. J. Context. Behav. Sci. 2015, 4, 1-11. [CrossRef]

55. Turrell, S.L.; Bell, M. ACT for Adolescents: Treating Teens and Adolescents in Individual and Group Therapy; New Harbinger Publications: Oakland, CA, USA, 2016.

56. Harris, R. ACT Made Simple; New Harbinger Publications: Oakland, CA, USA, 2009; pp. 9-13.

57. Strosahl, K.; Robinson, P.; Gustavsson, T. Brief Interventions for Radical Change: Principles and Practice of Focused Acceptance and Commitment Therapy; New Harbinger Publications: Oakland, CA, USA, 2012; ISBN 9781608823451/9781608823475/9781608823468.

58. Stoddard, J.A.; Afari, N. The Big Book of ACT Metaphors: A Practitioner's Guide to Experiential Exercises and Metaphors in Acceptance and Commitment Therapy; New Harbinger Publications: Oakland, CA, USA, 2014.

59. Villatte, J.L.; Vilardaga, R.; Villatte, M.; Plumb Vilardaga, J.C.; Atkins, D.C.; Hayes, S.C. Acceptance and Commitment Therapy modules: Differential impact on treatment processes and outcomes. Behav. Res. Ther. 2016, 77, 52-61. [CrossRef] [PubMed]

60. Cattivelli, R.; Castelnuovo, G.; Musetti, A.; Varallo, G.; Spatola, C.A.M.; Riboni, F.V.; Usubini, A.G.; Tosolin, F.; Manzoni, G.M.; Capodaglio, P.; et al. ACTonHEALTH study protocol: Promoting psychological flexibility with activity tracker and mHealth tools to foster healthful lifestyle for obesity and other chronic health conditions. Trials 2018, 19, 659. [CrossRef] [PubMed] 
61. Pietrabissa, G.; Castelnuovo, G.; Jackson, J.B.; Rossi, A.; Manzoni, G.M.; Gibson, P. Brief strategic therapy for bulimia nervosa and binge eating disorder: A clinical and research protocol. Front. Psychol. 2019, 10, 373. [CrossRef] [PubMed]

62. Faul, F.; Erdfelder, E.; Lang, A.G.; Buchner, A. G*Power 3: A flexible statistical power analysis program for the social, behavioral, and biomedical sciences. Behav. Res. Methods 2007, 39, 175-191. [CrossRef] [PubMed]

63. Cohen, J. Statistical Power Analysis for the Behavioral Sciences; Associates, L.E., Ed.; Lawrence Erlbaum Associates: Mahwah, NJ, USA, 1988.

64. The Jamovi Project. Jamovi (Version 1.6) [Computer Software]. 2021. Available online: https://www.jamovi.org (accessed on 4 June 2021).

65. Barlow, S.E. Expert committee recommendations regarding the prevention, assessment, and treatment of child and adolescent overweight and obesity: Summary report. Pediatrics 2007, 120 (Suppl. 4). [CrossRef]

66. Sanyaolu, A.; Okorie, C.; Qi, X.; Locke, J.; Rehman, S. Childhood and Adolescent Obesity in the United States: A Public Health Concern. Glob. Pediatr. Health 2019, 6. [CrossRef] [PubMed]

67. Aparicio, E.; Canals, J.; Arija, V.; De Henauw, S.; Michels, N. The role of emotion regulation in childhood obesity: Implications for prevention and treatment. Nutr. Res. Rev. 2016, 29, 17-29. [CrossRef] 